DIW BERLIN

Discussion

Papers

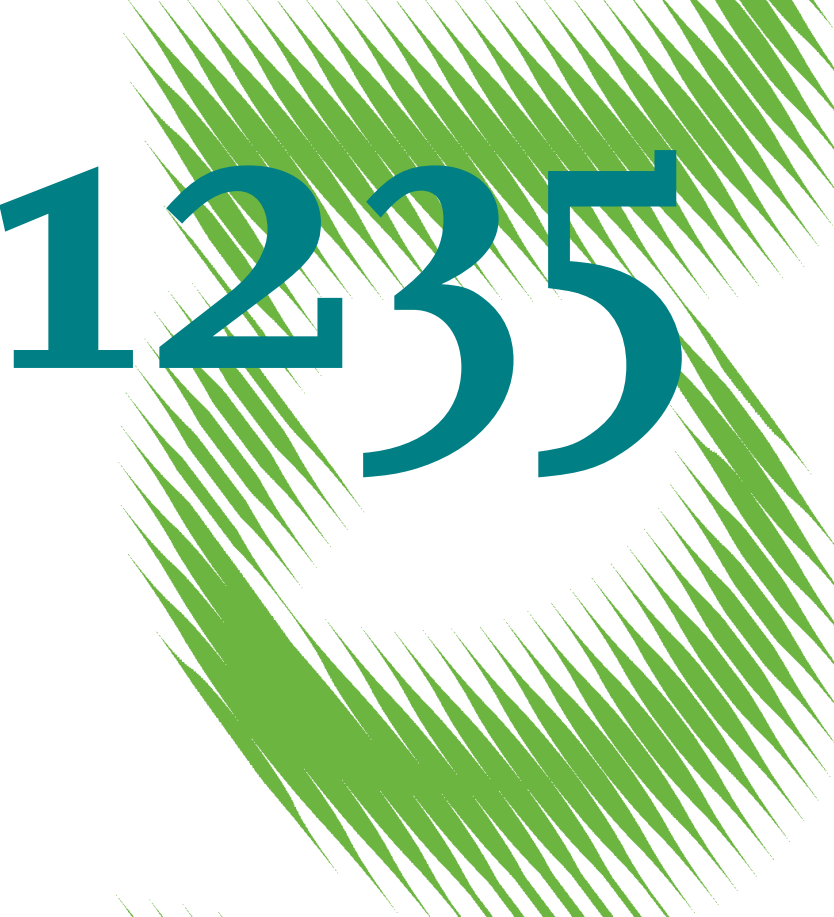

Reducing Confidence Bands for Simulated Impulse Responses 
Opinions expressed in this paper are those of the author(s) and do not necessarily reflect views of the institute.

IMPRESSUM

(C) DIW Berlin, 2012

DIW Berlin

German Institute for Economic Research

Mohrenstr. 58

10117 Berlin

Tel. $+49(30) 89789-0$

Fax +49 (30) $89789-200$

http://www.diw.de

ISSN print edition $1433-0210$

ISSN electronic edition 1619-4535

Papers can be downloaded free of charge from the DIW Berlin website:

http://www.diw.de/discussionpapers

Discussion Papers of DIW Berlin are indexed in RePEc and SSRN:

http://ideas.repec.org/s/diw/diwwpp.html

http://www.ssrn.com/link/DIW-Berlin-German-Inst-Econ-Res.html 


\title{
Reducing Confidence Bands for Simulated Impulse Responses
}

\author{
Helmut Lütkepoh $]^{1}$ \\ Freie Universität Berlin and DIW Berlin \\ Mohrenstr. 58, D-10117 Berlin, Germany \\ email: hluetkepohl@diw.de
}

August 1, 2012

\begin{abstract}
It is emphasized that the shocks in structural vector autoregressions are only identified up to sign and it is pointed out that this feature can result in very misleading confidence intervals for impulse responses if simulation methods such as Bayesian or bootstrap methods are used. The confidence intervals heavily depend on which variable is used for fixing the sign of the initial responses. In particular, when the shocks are identified via long-run restrictions the problem can be severe. It is pointed out that a suitable choice of variable for fixing the sign of the initial responses can result in substantial reductions in the confidence bands for impulse responses.
\end{abstract}

Key Words: Vector autoregressive process, impulse responses, bootstrap, Bayesian estimation

JEL classification: C32

${ }^{1}$ I thank Aleksei Netšunajev for comments on an earlier version of this paper. 


\section{Introduction}

Impulse responses are commonly used in structural vector autoregressive (SVAR) analysis in order to assess the responses of the variables to exogenous shocks. Confidence intervals around the impulse responses are typically generated by simulation methods. For example, the bootstrap is often used if classical methods are considered or simulations from the posterior distribution are performed if Bayesian inference is preferred. Such methods are usually conducted without accounting for the fact that the shocks are only locally identified. In this study it is pointed out that this approach can lead to very misleading confidence intervals because sampling may occur in different parts of the parameter space where likelihood values are identical.

Since conventional identification restrictions only identify the shocks up to sign, for each shock one of the variables is usually singled out for specifying or assigning the sign. For example, the sign of a monetary policy shock is often linked to the interest rate. If a contractionary monetary policy shock is of interest, the sign of the shock is chosen such that the interest rate increases on impact while for an expansionary shock the sign is fixed such that the initial response of the interest rate is negative. Unfortunately, the confidence intervals for impulse responses obtained by simulation methods typically depend on the variable used for sign-assignment and may in fact change dramatically if another variable is used.

To illustrate the problem, consider the bivariate $\operatorname{VAR}(1)$ process

$$
y_{t}=\left[\begin{array}{c}
\Delta q_{t} \\
U_{t}
\end{array}\right]=\nu+\left[\begin{array}{cc}
-0.1 & 0.8 \\
-0.4 & 1.0
\end{array}\right] y_{t-1}+u_{t} \quad \text { with } \quad \Sigma_{u}=\left[\begin{array}{cc}
0.73 & 0.12 \\
0.12 & 0.16
\end{array}\right],
$$

where $\nu=0$. Thinking of the first variable as being output growth (denoted by $\Delta q_{t}$ ) and the second component as unemployment rate (denoted as $U_{t}$ ), a Blanchard and Quah (1989) type identification assumes that there are supply $\left(\varepsilon_{t}^{s}\right)$ and demand $\left(\varepsilon_{t}^{d}\right)$ shocks and the latter do not have a long-run impact on output. Hence, the structural shocks are obtained from the reduced form residuals $u_{t}$ as $\varepsilon_{t}=\left(\varepsilon_{t}^{s}, \varepsilon_{t}^{d}\right)^{\prime}=B^{-1} u_{t}$, where the transformation matrix $B$ is identified (up to sign) by the long-run neutrality assumption for the demand shocks. I have generated time series of length $T=250$ using the data generation process (DGP) (1.1), estimated the parameters and simulated confidence intervals for the impulse responses by a bootstrap method using standard percentile intervals. The responses to a demand shock are displayed in Figure 1. On the left-hand side the responses to a shock, sign-assignment via the second variable, are shown. A positive demand shock has a negative 
effect on unemployment on impact, that is, a negative initial response of $U_{t}$ is imposed. In this case the confidence intervals of both impulse response functions are very wide and indicate insignificant responses of both variables to the shock. In sharp contrast, highly significant responses are obtained on the right-hand side of Figure 1 where the sign-assignment is done via the first variable $\left(\Delta q_{t}\right)$. More precisely, the shock is specified to have a positive instantaneous impact on $\Delta q_{t}$. Now the responses of both variables and their confidence intervals are quite plausible and quite different conclusions may be drawn from these responses than from those on the left-hand side of Figure 1 .

A word of caution may be in order at this point. When I speak of signassignment for a shock, that is, of identifying the shock by a sign restriction in this context, I only refer to the sign of the impact effect on a single variable. In fact, in most of the discussion in this paper the shocks are assumed to be identified by a set of equality restrictions. There is a literature on identification of shocks via sign restrictions that amounts to set or interval identification (see, e.g., Faust (1998), Canova and De Nicoló (2002), Uhlig (2005)). Although the problem discussed in the present study is also relevant in that context, as I will argue later, for the time being local identification of the shocks is assumed to be via equality restrictions, that is, I consider point identified shocks. Sign-assignment refers only to the question whether a positive or a negative shock is considered and that is specified via the initial response of a single variable.

It may be worth emphasizing that the problem considered in this study does not just occur when classical inference procedures are used but it is also present in Bayesian analysis. In this study, firstly, the problem will be investigated in greater detail and, secondly, a possible cure is proposed for those cases where it is likely to occur. It is suggested to use variables for sign-assignment with an initial response to the shock significantly away from zero. Alternatively, one may also do the sign-assignment on the basis of the long-run effects of a shock. It may also be a good idea to check the robustness of the confidence bands with respect to changes in the variable used for sign-assignment.

The remainder of the study is organized as follows. In Sec. 2 the model setup is presented. In Sec. 3 the problem is dissected in detail and solutions are proposed. Illustrative examples are discussed in Sec. 4 and Sec. 5 concludes. 

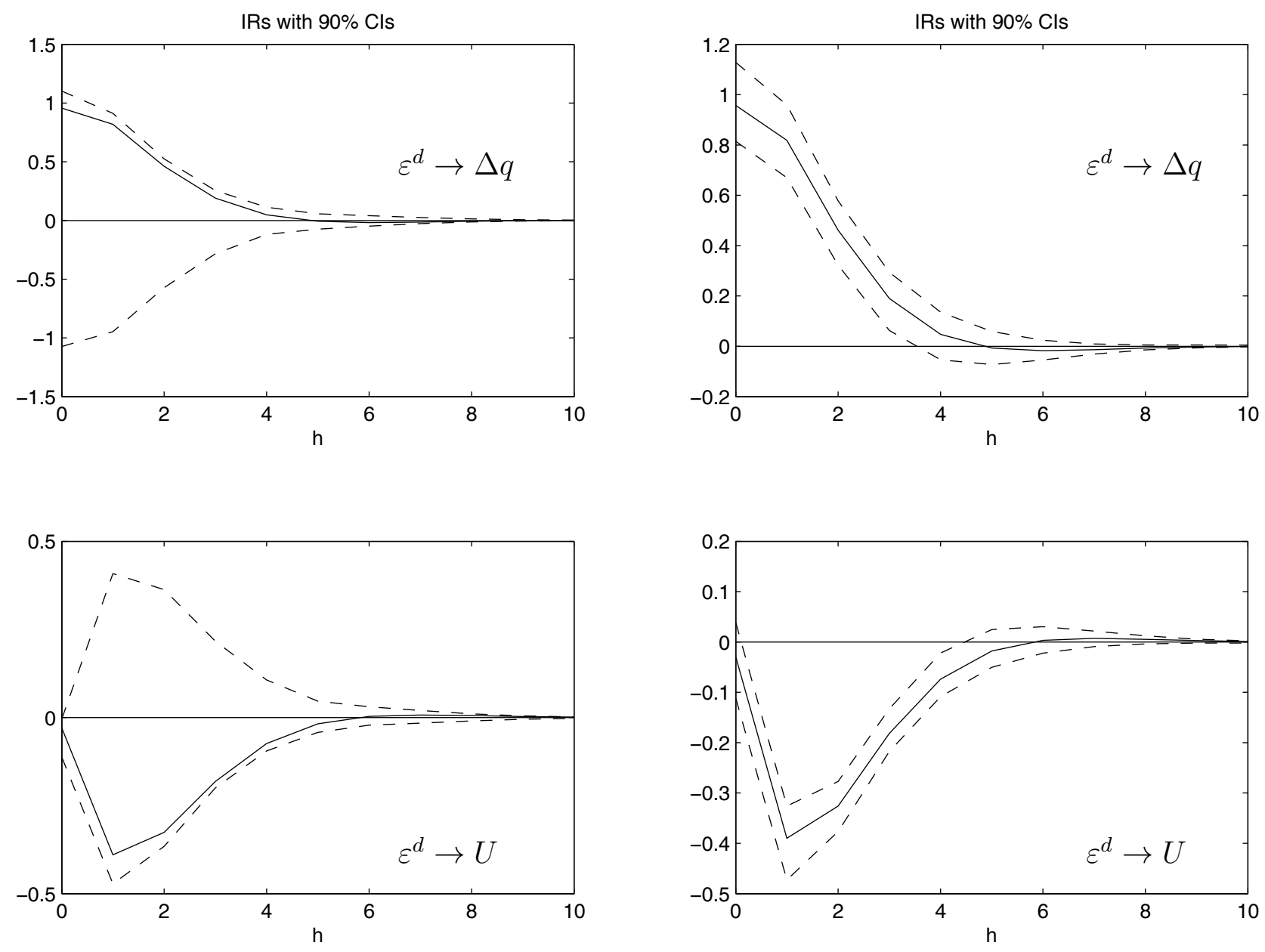

Figure 1: Responses to demand shock in example system with bootstrap confidence intervals based on 10000 replications (left-hand side: sign-assignment via $U_{t}$; right-hand side: sign-assignment via $\Delta q_{t}$ ). 


\section{Model Setup}

Let $y_{t}=\left(y_{1 t}, \ldots, y_{K t}\right)^{\prime}$ be generated by a $K$-dimensional $\operatorname{VAR}(p)$ process,

$$
y_{t}=A_{1} y_{t-1}+\cdots+A_{p} y_{t-p}+u_{t},
$$

where the $A_{i}(i=1, \ldots, p)$ are $(K \times K)$ parameter matrices and the error process $u_{t}=\left(u_{1 t}, \ldots, u_{K t}\right)^{\prime}$ is a $K$-dimensional zero mean white noise process with covariance matrix $E\left(u_{t} u_{t}^{\prime}\right)=\Sigma_{u}$, that is, $u_{t} \sim\left(0, \Sigma_{u}\right)$. There are no deterministic terms because they are not relevant for the problem of interest in the following. Adding them does not change the results. Of course, in practice such terms will have to be included.

Using the lag operator and defining the matrix polynomial in the lag operator $A(L)$ as $A(L)=I_{K}-A_{1} L-\cdots-A_{p} L^{p}$, the process (2.1) can be written as

$$
A(L) y_{t}=u_{t}
$$

The process is stable if

$$
\operatorname{det} A(z)=\operatorname{det}\left(I_{K}-A_{1} z-\cdots-A_{p} z^{p}\right) \neq 0 \text { for } z \in \mathbb{C},|z| \leq 1 .
$$

The representation $(2.1) / 2.2$ is a reduced form because all variables on the right-hand side are predetermined at time $t$. In contrast, structural form models may have contemporaneous variables as explanatory variables in some equations, as in

$$
A_{0} y_{t}=A_{1}^{*} y_{t-1}+\cdots+A_{p}^{*} y_{t-p}+\varepsilon_{t} .
$$

Here the $(K \times K)$ matrix $A_{0}$ represents the instantaneous relations and $A_{j}^{*}=$ $A_{0} A_{j}(j=1, \ldots, p)$. The structural form error term $\varepsilon_{t}=A_{0} u_{t}$ is a white noise process with covariance matrix $\Sigma_{\varepsilon}=A_{0} \Sigma_{u} A_{0}^{\prime}$. The main diagonal of the matrix $A_{0}$ usually consists of ones. In that case the set of equations in (2.4) can be written such that each of the variables appears on the left-hand side of one of the equations and may depend on contemporaneous values of some or all of the other variables. The covariance matrix $\Sigma_{\varepsilon}$ is typically a diagonal matrix, that is, the structural errors are contemporaneously uncorrelated (sometimes referred to as orthogonal).

It is often helpful to think of specific shocks hitting the system and hence, specifying the structure not through the instantaneous relations of the observed variables but via the instantaneous effects of the shocks. For that case a suitable structural model setup is

$$
y_{t}=A_{1} y_{t-1}+\cdots+A_{p} y_{t-p}+B \varepsilon_{t},
$$


where $B$ is the matrix of instantaneous effects. In this setup it is, for instance, easy to specify that a certain structural shock $\varepsilon_{i t}$ does not have an instantaneous effect on one of the observed variables by restricting the corresponding element of $B=A_{0}^{-1}$ to be zero. Other restrictions are also possible and will be discussed in the next section. Given the formal equivalence of the $A$ - and $B$-models, I focus on the latter for simplicity in the following.

\section{$3 \quad$ Simulating Impulse Responses}

Although equality and inequality constraints may be used for identifying shocks in structural VAR models, I focus on the case of equality constraints in the following because the problem is especially severe for this type of identifying restrictions. Since $B$ is the matrix of instantaneous effects of the structural shocks, equality constraints on $B$ sometimes come in the form of zero constraints on its elements, that is,

$$
R \operatorname{vec}(B)=0
$$

where $R$ is a suitable selection matrix. For example, recursively identified models with a triangular $B$ matrix are considered by Sims (1980), Christiano, Eichenbaum and Evans (1996), Sims, Waggoner and Zha (2008), Kilian (2009) and many others. Alternatively, zero restrictions are often imposed on the long-run effects of the shocks as in Blanchard and Quah (1989),

$$
R_{l} \operatorname{vec}\left(A(1)^{-1} B\right)=0,
$$

where $R_{l}$ is a suitable selection matrix, or, if there is cointegration, as in King, Plosser, Stock and Watson (1991),

$$
R_{l} \operatorname{vec}(\Xi B)=0,
$$

where $\Xi$ is the matrix of reduced-form long-run effects given, e.g., by Lütkepohl (2005, Sec. 9.2). Using standard rules of the vec operator, the last two sets of restrictions can also be written as in (3.1) by defining $R=R_{l}\left(I_{K} \otimes A(1)^{-1}\right)$ and $R=R_{l}\left(I_{K} \otimes \Xi\right)$, respectively. Thus, the full set of restrictions for $B$ is

$$
B B^{\prime}=\Sigma_{u} \quad \text { and } \quad R \operatorname{vec}(B)=0,
$$

where $R$ is now a general restriction matrix.

From this set of restrictions for $B$, it is easily seen that the matrix is only identified up to sign (i.e., locally) at best because, multiplying any column of $B$ by -1 , the equations will still hold. Suppose that the restrictions indeed locally identify $B$, that is, they identify $B$ up to changes in sign for 
the columns. This is enough for asymptotic and Bayesian inference because changing all signs in a column of $B$ just means that negative instead of positive shocks are considered or vice versa. The user of a SVAR model will usually know which shocks s/he is interested in and consider the corresponding impulse responses. In fact, it is typically assumed that the $i$ th shock has a positive (or negative) effect on the $i$ th variable. This may indeed be no problem if there are only zero restrictions on the instantaneous effects, as in (3.1) and the shocks are somehow linked to the observed variables in the system. For example, if the last variable is an interest rate, the monetary shock is often specified to be the last element in the vector of structural shocks, $\varepsilon_{t}$. Then by a contractionary monetary policy shock we usually mean an increase in the interest rate and hence a positive initial effect of the last element in $\varepsilon_{t}$ on the last variable in the system.

However, the association between the shocks and the variables or their reactions is not always so clear. Consider for example the original Blanchard and Quah (1989) model that consists of an unemployment rate $U_{t}$ and output growth, $\Delta q_{t}$, with $q_{t}$ being log output and $\Delta$ the differencing operator. They considered a supply shock and a demand shock and identified the shocks by restricting the long-run effect of the demand shock on output to be zero. In other words, defining $y_{t}=\left(\Delta q_{t}, U_{t}\right)^{\prime}$ and $\varepsilon_{t}=\left(\varepsilon_{t}^{s}, \varepsilon_{t}^{d}\right)^{\prime}$,

$$
A(1)^{-1} B=\left(\begin{array}{ll}
* & 0 \\
* & *
\end{array}\right)
$$

where an asterisk denotes an unrestricted element. Note that the responses of $q_{t}$ are the accumulated responses of $\Delta q_{t}$. Thus, no long-run response of $q_{t}$ means a zero accumulated effect on $\Delta q_{t}$. In this example, the labels of the shocks are not directly linked to the observable variables of the system. Still, the specification of the signs of the shocks requires a decision on their effects on the variables. Thus, one may identify the signs of the columns of $B$ by assuming that the first variable reacts positively to a demand shock on impact and the second variable is reduced on impact when a supply shock hits. Of course, it is possible that the actual instantaneous responses are close to zero in which case this type of sign-assignment may cause problems because the estimated reaction may not have the same sign as the actual reaction and the instantaneous effect on the other variable may change sign if the sign of the instantaneous response of one variable is fixed in all simulations.

Again, I emphasize that sign-assignment in the context of this discussion is different from the related literature on identification via sign restrictions. In the present context the shocks are point identified via equality restrictions as in (3.4) whereas in the literature on sign restrictions à la Uhlig (2005) and others the shocks or parameters are only set-identified. 
Returning to the present setup, the problem can be stated more formally as follows. Let $b$ be the $i$ th column of $B$, that is, $b$ represents the vector of instantaneous effects of the $i$ th shock. The sign-assignment should always be done for an element that is clearly distinct from zero because then enforcing a particular sign is not restrictive for the other elements. If sign-assignment is done via an element that is close to zero, this may lead to distorted simulated confidence intervals of the other parameters in $b$. Suppose $b=\left(b_{1}, b_{2}\right)^{\prime}$ is just 2 -dimensional and sign-assignment is done on $b_{2}$, although the true value of $b_{2}=0$. Suppose further that the actual distribution of the estimator $\hat{b}$ is $\mathcal{N}\left(b, \Sigma_{b}\right)$. A valid $1-\gamma$ confidence interval for $b_{1}$ would be

$$
\left[\hat{b}_{1}-c_{1-\gamma / 2} \sigma_{11}, \hat{b}_{1}+c_{1-\gamma / 2} \sigma_{11}\right]
$$

where $c_{\eta}$ is the $\eta$ quantile of the standard normal distribution and $\sigma_{11}^{2}$ is the upper left hand element of $\Sigma_{b}$. However, if we choose the confidence interval by the following simulation method, a quite different, much wider interval may be obtained. Get $b^{(n)}$ for $n=1, \ldots, N$ as follows:

1. Draw $\tilde{b}=\left(\tilde{b}_{1}, \tilde{b}_{2}\right)^{\prime}$ from the $\mathcal{N}\left(b, \Sigma_{b}\right)$ distribution.

2. Set

$$
b^{(n)}=\left\{\begin{array}{rll}
\tilde{b} & \text { if } & \tilde{b}_{2} \geq 0 \\
-\tilde{b} & \text { if } & \tilde{b}_{2}<0
\end{array}\right.
$$

Choosing the confidence interval for $b_{1}$ from the empirical distribution as

$$
\left[b_{1}^{(N \gamma / 2)}, b_{1}^{(N(1-\gamma / 2))}\right]
$$

can obviously result in a much wider confidence interval with a not much larger inclusion probability because the sign of $\hat{b}_{1}$ is flipped around with probability one half even if the true $b_{1}$ is clearly and significantly positive. The actual coverage probability and length of the interval (3.7) depends, of course, on the true parameter values. The problem is that $b_{2}$ will take positive and negative values in the simulations. Whenever a negative value occurs, it will be converted to a positive value by the above sign convention. At the same time $b_{1}$ will also be reflected to the other side of the origin. Effectively, a pair $\left(b_{1}, b_{2}\right)^{\prime}$ from a part of the parameter space associated with a different local maximum of the likelihood function is chosen in this way. When $b_{1}$ is always positive in the area of the parameter space corresponding to the first optimum of the likelihood function this can result in a very wide confidence interval for $b_{1}$ because $b_{1}$ values from two different local optima of the likelihood function are included, even if the area of variation of $b_{1}$ is small in the 
neighborhood of one local optimum. If $b_{1}$ had been used for sign-assignment, the situation would be quite different. These considerations suggest that it is a good strategy to avoid sign-assignment based on imprecisely estimated impact effects. In other words, one may want to check the $t$-ratios of the estimated elements of $B$ and consider variables for sign-assignment that have impact effects with large (in absolute value) $t$-ratios.

In the following section the problem is illustrated with two examples from the literature. Before that it may be worth mentioning that I have discussed the problem in terms of the usual percentile interval that is typically considered in the impulse response literature. The same problem arises, of course, if small-sample corrections, as discussed by Kilian (1998), or the confidence intervals referred to as Hall intervals by Benkwitz, Lütkepohl and Wolters (2001) are considered.

\section{Illustrative Examples}

Two examples from the literature are considered in the following in order to illustrate the problem in a real-life setting. The examples are not chosen so as to criticize previous studies but rather to illustrate the problem that the choice of variable used for sign-assignment of a shock can be severe. The first example considers the Blanchard and Quah (1989) model, while the second is based on a model for the Canadian labor market from Breitung, Brüggemann and Lütkepohl (2004).

\subsection{Blanchard-Quah Model}

Blanchard and Quah (1989) consider a model for $y_{t}=\left(\Delta q_{t}, U_{t}\right)^{\prime}$ based on quarterly U.S. data from 1948Q2 - 1987Q4.2 Output $q_{t}$ is measured as the log of gross domestic product and $U_{t}$ is the unemployment rate. I use a $\operatorname{VAR}(8)$ model with intercept for $y_{t}$, as in Blanchard and Quah (1989). Identification of a supply and a demand shock $\left(\varepsilon_{t}^{s}, \varepsilon_{t}^{d}\right)^{\prime}$ is done via the assumption that the demand shock has no long-run impact on output as in (3.5).

Before turning to the impulse response analysis, it may be worth emphasizing that the methods considered here rely on structural stability of the model throughout the sample period. The individual equations of the present model pass the CUSUM but not the CUSUM-of-squares test for structural change at a $5 \%$ level of significance. The CUSUM test was shown to be valid for dynamic models with lagged dependent variables by Krämer,

\footnotetext{
${ }^{2}$ The data used in the following are from the archive of the Journal of Applied Econometrics for the article Weber (1995).
} 
Ploberger and Alt (1988). They also show that the CUSUM test may be preferable to the CUSUM-of-squares test in terms of power (see also Krämer and Sonnberger (1986), Ploberger, Krämer and Kontrus (1989) and Ploberger and Krämer (1992) for further discussion of these and other stability tests). We ignore possible instability problems because the main objective of using this example from the literature is to illustrate the theoretical issues discussed in the previous section and not to question the specification of models used by other authors.

In Figure 2 impulse responses with $90 \%$ confidence intervals are presented. In the upper part of the figure the supply shock is assumed to have a nonnegative effect on impact on output growth and the demand shock is assumed to decrease unemployment on impact whereas in the lower part of the figure $\varepsilon_{t}^{s}\left(\varepsilon_{t}^{d}\right)$ has a positive instantaneous effect on $U_{t}\left(q_{t}\right)$. Clearly the size of the confidence bands for the impulse responses due to a supply shock changes dramatically with the choice of variable for sign-assignment whereas the confidence bands for the responses to demand shocks are not much affected by the variable used for sign-assignment. The reason is seen in Figure 2. The output response on impact to a supply shock is close to zero. Therefore, specifying the sign of the shock on the basis of the output response results in many sign changes in the bootstrap that are reflected in the wide confidence intervals for both the responses of output and unemployment to a supply shock in the upper part of Figure 2. In fact, based on these confidence bands one may conclude that neither output nor unemployment respond significantly to a supply shock.

Since the unemployment response is clearly and significantly positive, the situation changes dramatically in the lower part of Figure 2 where the confidence bands are much narrower. Now significant responses of both variables may be diagnosed. Also, on the left-hand side of Figure 2 where the responses to a demand shock are displayed, the situation is very different. Since both variables react significantly to a demand shock on impact, it does not matter which variable is used for sign-assignment. Hence, the example clearly illustrates the point that normalizing the sign on a variable with little or no response on impact may be problematic and that variables with strong and significant impact responses should be used for this purpose.

\subsection{Canadian Labor Market Model}

In the second example I consider a quarterly labor market model for Canada that was analyzed by Breitung et al. (2004). These authors use four variables. Specifically, $g d p_{t}, e_{t}, U_{t}$ and $w_{t}$ denote the $\log$ of real gross domestic product, the log of employment, the unemployment rate and the log of a 

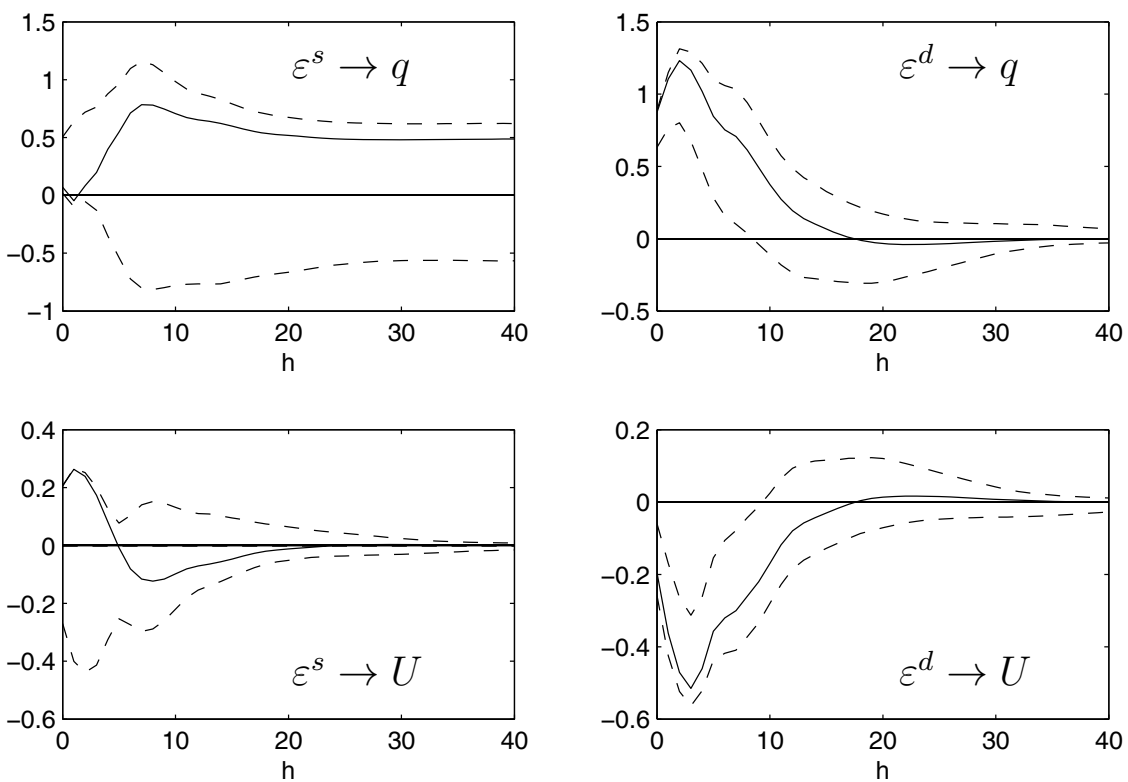

(sign-assignment based on $a$ on the left and on $U$ on the right)
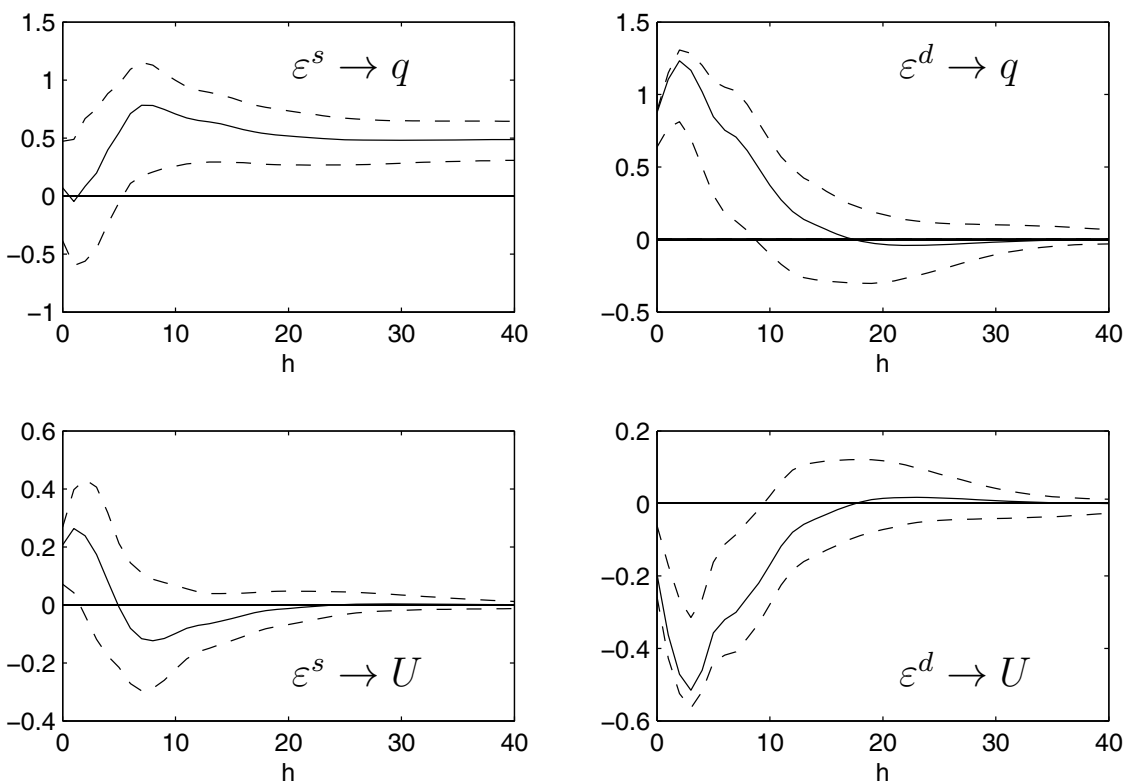

(sign-assignment based on $U$ on the left and on $q$ on the right)

Figure 2: Responses to supply and demand shocks in Blanchard-Quah system with $90 \%$ bootstrap confidence intervals based on 10000 replications. 
real wage index, respectively. The vector $y_{t}=\left(g d p_{t}-e_{t}, e_{t}, U_{t}, w_{t}\right)^{\prime}$ includes the variables productivity, employment, unemployment and real wages. Breitung et al. (2004) use quarterly, seasonally adjusted data for Canada from the OECD database for the period 1980Q1 - 2000Q4.3 They consider technology, labor demand, labor supply and wage-setting shocks, denoted as $\varepsilon_{t}=\left(\varepsilon_{t}^{g d p}, \varepsilon_{t}^{d}, \varepsilon_{t}^{s}, \varepsilon_{t}^{w}\right)^{\prime}$.

The identification approach follows King et al. (1991). Breitung et al. (2004) use a VAR(3) model with trend and find one cointegration relation. They assume that there is one transitory shock (the wage-setting shock) and three shocks with permanent effects $\left(\varepsilon_{t}^{g d p}, \varepsilon_{t}^{d}, \varepsilon_{t}^{s}\right)$. Identification is done via the following exclusion restrictions for the instantaneous and long-run effects:

$$
B=\left[\begin{array}{cccc}
* & * & * & * \\
* & * & * & * \\
* & * & * & * \\
* & 0 & * & *
\end{array}\right] \text { and } \Xi B=\left[\begin{array}{cccc}
* & 0 & 0 & 0 \\
* & * & * & 0 \\
* & * & * & 0 \\
* & * & * & 0
\end{array}\right]
$$

As before, $B$ denotes the matrix of instantaneous effects, $\Xi B$ is the matrix of long-run effects of the shocks and asterisks denote unrestricted elements. Thus, demand and supply shocks are assumed to have no long-run effects on productivity and wage-setting shocks do not have a permanent impact on any of the variables in $y_{t}$. Since these restrictions are not sufficient to identify all four shocks, a further restriction is imposed on the impact effects. More precisely, Breitung et al. (2004) assume that labor demand shocks do not have an instantaneous effect on wages although they admit that such a restriction is not suggested by economic theory in their model setup. The restriction is imposed because the authors need a further restriction to just-identify the four shocks.

For sign-assignment of the shocks it is useful to take into account the expected relation of the shocks to the variables as well as the estimated instantaneous effects matrix. The following estimated matrix is reported by Breitung et al. (2004):

$$
\tilde{B}=\left[\begin{array}{rrrr}
0.62 & 0.08 & -0.16 & 0.07 \\
(5.98) & (0.64) & (-0.66) & (0.92) \\
-0.13 & 0.28 & -0.17 & 0.10 \\
(-1.69) & (4.11) & (-0.87) & (2.21) \\
0.03 & -0.29 & 0.01 & 0.05 \\
(0.44) & (-5.45) & (0.09) & (1.59) \\
0.12 & 0 & 0.52 & 0.52 \\
(0.74) & & (0.74) & (5.91)
\end{array}\right]
$$

\footnotetext{
${ }^{3}$ The data are available in the JMulTi database, see Lütkepohl and Krätzig (2004), and can be downloaded from http://www.jmulti.com/.
} 
where $t$-ratios obtained by a bootstrap procedure are shown in parentheses under the maximum likelihood estimates. A positive technology shock is expected to increase productivity while its immediate impact on the other variables is less clear. Hence, the sign-assignment is done via the first variable, $g d p_{t}-e_{t}$. This choice should work well given that the $t$-value of the upper left-hand element in $B$ is quite high (5.98) and, hence, the estimated instantaneous effect of the technology shock on productivity is well away from zero and highly significant. A positive labor demand shock is expected to increase employment and reduce the unemployment rate so that these two variables are candidates for sign-assignment of the labor demand shock. Since the two corresponding elements in the estimated $B$ matrix are both highly significant, any of these variables could be chosen for sign-assignment for the labor demand shock and the choice should not make much difference for the confidence bands of the impulse responses. Also the sign of the wagesetting shock is naturally assigned via its impact on the wage and indeed the estimated impact effect on the real wage is highly significant so that there should not be a problem using this variable for sign-assignment of the wage-setting shock.

The situation is quite different for the labor supply shock. Its impact effects are seen in the third column of $\tilde{B}$. A positive shock might be expected to increase the unemployment rate on impact and perhaps reduce wages. Otherwise the implications are not clear and, in fact, none of the elements in the third column of the impact matrix $B$ are significant at usual levels. All $t$-ratios are smaller than one in absolute value. Hence, it is not clear which variable should be used for sign-assignment of the labor supply shock and one may expect that the choice matters for the size of the confidence intervals around the impulse responses.

Since the reaction of unemployment to the supply shock is of particular interest, the relevant impulse responses are shown in Figure 3 . In the upper half the responses and confidence intervals are based on sign-assignment via the impact effect on $U_{t}$ on the left and on $w_{t}$ on the right. In both cases the confidence intervals are very wide and always include the zero line. If the signassignment is via the wage response, the response of the unemployment rate even becomes negative, that is, one may get the impression that a positive labor supply shock reduces unemployment. Of course, the result is not at all significant and such a conclusion is not likely to be drawn from the figure. Still, the graphs show the impact of the choice of the variable used for signassignment.

In that situation one may also consider doing the sign-assignment of the shock via its long-term effects. In other words, one may enforce a particular sign of the elements of the long-run effects matrix $\Xi B$. Of course, one would 

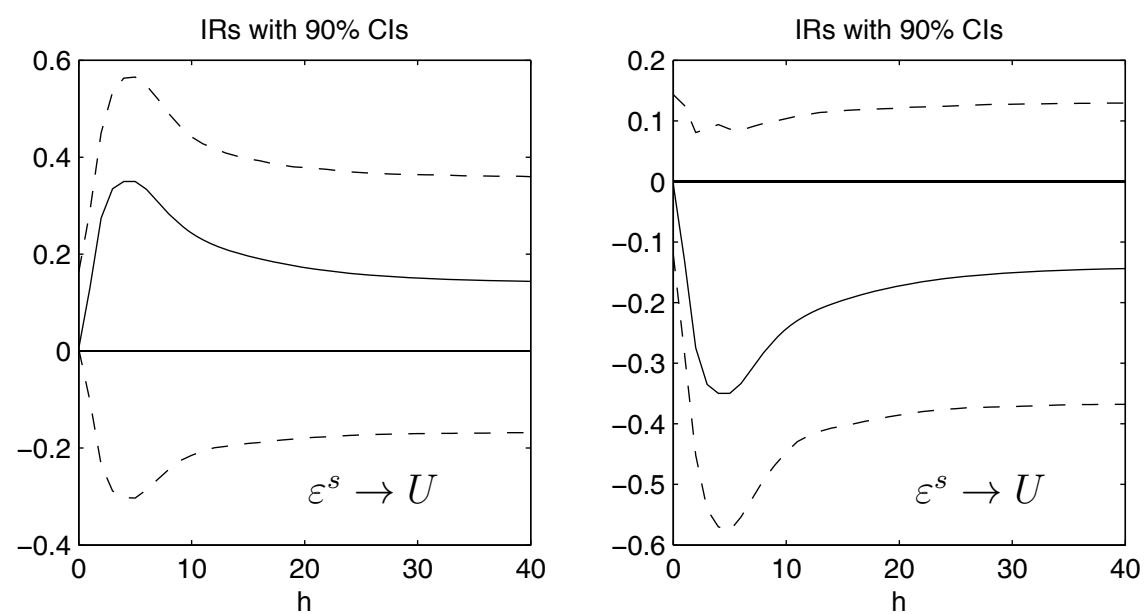

(sign-assignment via impact effect on $U_{t}$ on the left and on $w_{t}$ on the right)
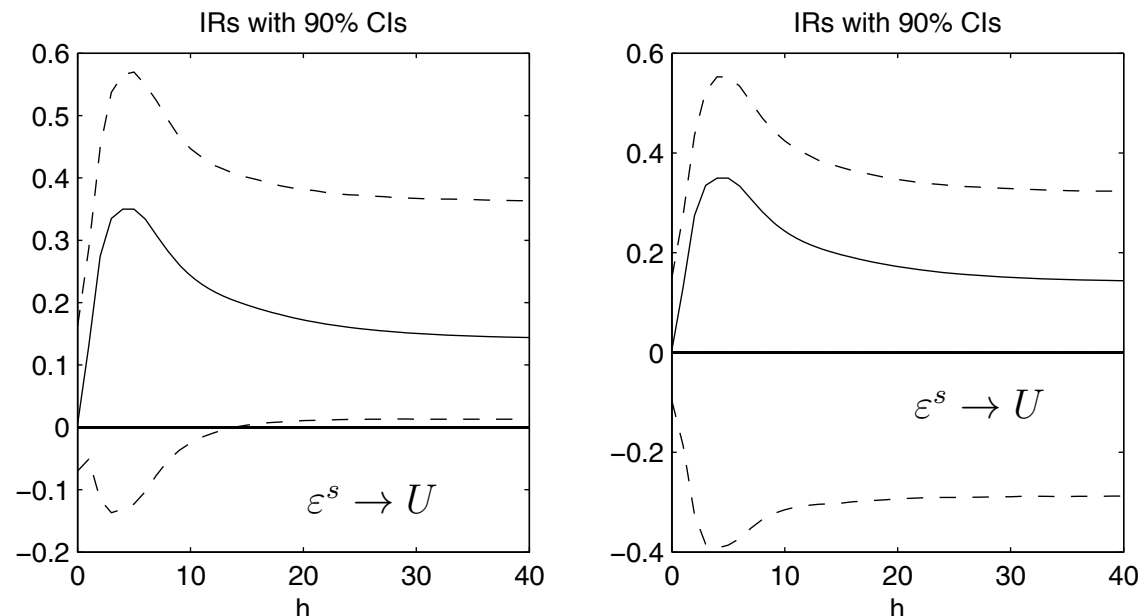

(sign-assignment via long-run effect on $U_{t}$ on the left and on $w_{t}$ on the right)

Figure 3: Unemployment rate responses to supply shocks in the Canadian model with $90 \%$ bootstrap confidence intervals based on 10000 replications. 
also expect a labor supply shock to increase unemployment and reduce real wages in the long-run, if there is a long-run effect at all. In the lower half of Figure 3 I show the impulse responses of the unemployment rate to a supply shock if the sign-assignment is based on the long-run effects on the unemployment rate (on the left) and on wages (on the right). Again they are very different. If the long-run effect of $w_{t}$ are used, the confidence intervals are again very wide and include zero for all lead times. In contrast, if the sign-assignment is done via the long-run response of the unemployment rate, the short-run responses of $U_{t}$ remain insignificant but the intervals around the longer-horizon responses do not include zero and, hence, indicate a significant longer-term increase in the unemployment rate. Thus, this example again illustrates the dramatic effects of sign-assignment on the size of the confidence intervals around the impulse responses. The general message of these illustrations is that it can be beneficial to consider different options for sign-assignment of a shock. It can help to consider highly significant effects for sign-assignment and to avoid imprecisely estimated impact effects. Doing this can lead to substantial reductions in the confidence intervals of impulse responses if the intervals are generated by simulation methods.

\section{Conclusions}

In this study it is pointed out that although in SVAR analysis the sign of a structural shock is usually chosen in a casual fashion, it may be worth spending some effort in fixing the sign. In particular, it is argued and demonstrated that if the confidence intervals for impulse responses are determined by simulation techniques such as the bootstrap or Bayesian procedures the size of the intervals can be reduced substantially by a suitable choice of the variable used for assigning the sign of the shock. For example, in a Canadian labor market model the confidence intervals around the impulse responses of the unemployment rate to a labor supply shock where shown to be very different depending on the variable used for sign-assignment of the shock. Thus, it can be beneficial to consider different possibilities for sign-assignment of the shocks in a SVAR analysis. Specifying the sign on the basis of the instantaneous effects or their long-run effects on the variables may be considered and it is recommended to avoid sign-assignment via imprecisely estimated effects.

In this study the discussion of the problem and its solutions have been discussed in the framework of exact equality restrictions for the identification of the structural shocks. It may be worth pointing out that the problem may also come up if sign restrictions are used for identification rather than con- 
ventional equality constraints. Note that identification via sign restrictions has to be separated from the choice of positive or negative shocks. However, when sign restrictions are used for identification, the problem may be less severe because the investigator has given some thought to the sign of the responses to the shocks and may have imposed sufficient restrictions to fix the initial responses of all the variables. If the sign-restrictions leave room for choice, then the problem described in the foregoing may also be present and the solutions proposed in the present study are also available in the framework of identification via sign restrictions.

\section{References}

Benkwitz, A., Lütkepohl, H. and Wolters, J. (2001). Comparison of bootstrap confidence intervals for impulse responses of German monetary systems, Macroeconomic Dynamics 5: 81-100.

Blanchard, O. and Quah, D. (1989). The dynamic effects of aggregate demand and supply disturbances, American Economic Review 79: 655-673.

Breitung, J., Brüggemann, R. and Lütkepohl, H. (2004). Structural vector autoregressive modeling and impulse responses, in H. Lütkepohl and M. Krätzig (eds), Applied Time Series Econometrics, Cambridge University Press, Cambridge, pp. 159-196.

Canova, F. and De Nicoló, G. (2002). Monetary disturbances matter for business fluctuations in the G-7, Journal of Monetary Economics 49: 1131-1159.

Christiano, L. J., Eichenbaum, M. and Evans, C. (1996). The effects of monetary policy shocks: Evidence from the flow of funds, Review of Economics and Statistics 78: 16-34.

Faust, J. (1998). The robustness of identified VAR conclusions about money, Carnegie-Rochester Conference Series in Public Policy 49: 207-244.

Kilian, L. (1998). Small-sample confidence intervals for impulse response functions, Review of Economics and Statistics 80: 218-230.

Kilian, L. (2009). Not all oil price shocks are alike: Disentangling demand and supply shocks in the crude oil market, American Economic Review 99: 10531069.

King, R. G., Plosser, C. I., Stock, J. H. and Watson, M. W. (1991). Stochastic trends and economic fluctuations, American Economic Review 81: 819-840.

Krämer, W., Ploberger, W. and Alt, R. (1988). Testing for structural change in dynamic models, Econometrica 56: 1355-1369. 
Krämer, W. and Sonnberger, H. (1986). The Linear Regression Model Under Test, Physica-Verlag, Heidelberg.

Lütkepohl, H. (2005). New Introduction to Multiple Time Series Analysis, Springer-Verlag, Berlin.

Lütkepohl, H. and Krätzig, M. (eds) (2004). Applied Time Series Econometrics, Cambridge University Press, Cambridge.

Ploberger, W. and Krämer, W. (1992). The CUSUM test with OLS residuals, Econometrica 60: 271-285.

Ploberger, W., Krämer, W. and Kontrus, K. (1989). A new test for structural stability in the linear regression model, Journal of Econometrics 40: 307318.

Sims, C. A. (1980). Macroeconomics and reality, Econometrica 48: 1-48.

Sims, C. A., Waggoner, D. F. and Zha, T. (2008). Methods for inference in large multiple-equation Markov-switching models, Journal of Econometrics 146: $255-274$.

Uhlig, H. (2005). What are the effects of monetary policy on output? Results from an agnostic identification procedure, Journal of Monetary Economics 52: $381-419$

Weber, C. E. (1995). Cyclical output, cyclical unemployment, and Okun's coefficient: A new approach, Journal of Applied Econometrics 10: 433-445. 